\title{
A GROWTH THEOREM FOR BIHOLOMORPHIC MAPPINGS ON A BANACH SPACE
}

\author{
Tatsuhiro Honda
}

\begin{abstract}
Let $\|\cdot\|$ be an arbitrary norm on a Banach space $E$. Let $B$ be the open unit ball of $E$ for the norm $\|\cdot\|$, and let $f: B \rightarrow E$ be a biholomorphic convex mapping such that $f(0)=0$ and $d f(0)$ is identity. We will give an upper bound of the growth of $f$.
\end{abstract}

\section{Introduction}

Let $\Delta=\{z \in \mathbb{C}:|z|<1\}$ denote the open unit disc in the complex plane $\mathbb{C}$. Let $f: \Delta \rightarrow \mathbb{C}$ be a biholomorphic convex mapping with $f(0)=0$ and $f^{\prime}(0)=1$. Then the following inequality holds :

$$
|f(z)| \leq \frac{|z|}{1-|z|}, \text { for all } z \in \Delta .
$$

It is natural to consider a generalization of the growth theorem above to $\mathbb{C}^{n}$. Let $\Omega$ be a domain in $\mathbb{C}^{n}$ which contains the origin in $\mathbb{C}^{n}$. A holomorphic mapping $f: \Omega \rightarrow \mathbb{C}^{n}$ is said to be normalized, if $f(0)=0$ and the Jacobian matrix $D f(0)$ at the origin is identity. Let $\mathbb{B}^{n}$ denote the Euclidean unit ball in $\mathbb{C}^{n}$. Let $f: \mathbb{B}^{n} \rightarrow \mathbb{C}^{n}$ be a normalized biholomorphic convex mapping. Then C. H. FitzGerald and C. R. Thomas [4], T. Liu [11] and T. J. Suffridge [12] extended the upper bound above for the growth of $f$ to $\mathbb{B}^{n}$ in $\mathbb{C}^{n}$ by using different methods and showed that

$$
\|f(z)\|_{2} \leq \frac{\|z\|_{2}}{1-\|z\|_{2}} \text { for all } z \in \mathbb{B}^{n}
$$

1991 Mathematics Subject Classification. Primary 32H02. Secondary $30 \mathrm{C} 45$. 
where $\|\cdot\|_{2}$ denotes the Euclidean norm. Let

$$
B_{p}=\left\{z=\left(z_{1}, \ldots, z_{n}\right) \in \mathbb{C}^{n}:\|z\|_{p}=\left(\sum_{i=1}^{n}\left|z_{i}\right|^{p}\right)^{1 / p}<1\right\}
$$

for $p \geq 1$ and

$D\left(p_{1}, \ldots, p_{n}\right)=\left\{z=\left(z_{1}, \ldots, z_{n}\right) \in \mathbb{C}^{n}:\left|z_{1}\right|^{p_{1}}+\cdots+\left|z_{n}\right|^{p_{n}}<1\right\}$

with $p_{1}, \ldots, p_{n} \geq 1$. S. Gong and T. Liu [7] gave the upper bound above for the growth of normalized biholomorphic convex mappings on $B_{p}$ and $D\left(p_{1}, \ldots, p_{n}\right)$ and H. Hamada [8] proved a similar result on the unit ball in $\mathbb{C}^{n}$ with respect to an arbitrary norm. In this paper, we give the upper bound for the growth of a biholomorphic convex mapping on the unit ball in a complex Banach space as follows.

Main Theorem Let $E$ be a complex Banach space with norm $\|\cdot\|$. Let $B$ be the open unit ball of $E$ for the norm $\|\cdot\|$, and let $f: B \rightarrow E$ be a biholomorphic convex mapping such that $f(0)=0$ and $d f(0)$ is identity. Then

$$
\|f(z)\| \leq \frac{\|z\|}{1-\|z\|}
$$

for all $z \in B$.

\section{Notation and preliminaries}

Let $U$ be an open set in a complex normed space $E$ and let $F$ be a complex Banach space. Let $f$ be a holomorphic mapping from $U$ to $F$. Then the following equation holds in a neighbourhood $V$ of $x$ in $U$ for $x \in U$ :

$$
f(z)=\sum_{n=1}^{\infty} P_{n}(z-x)
$$

where

$$
P_{n}(y)=\frac{d^{n} f(x)}{n !}(y)=\frac{1}{2 \pi \sqrt{-1}} \int_{|\zeta|=1} \frac{1}{\zeta^{n+1}} f(x+\zeta y) d \zeta
$$


for any $y \in E \backslash\{0\}$ such that $x+\zeta y \in U$ for all $\zeta \in \mathbb{C}$ with $|\zeta| \leq 1$. The series (2.1) is called the Taylor expansion of $f$ by $n$-homogeneous polynomials $P_{n}$ at $x$.

Let $E$ be a complex Banach space with norm $\|\cdot\|$. Let $B$ be the open unit ball of $E$ for the norm $\|\cdot\|$, and let $f: B \rightarrow E$ be a biholomorphic mapping. A biholomorphic mapping $f$ is said to be convex if $f(B)$ is convex.

The following theorem (the Maximum Modulus Principle) is well-known (see, for example, Dunford and Schwartz [3]).

Theorem 2.1 Let $E$ be a complex Banach space with norm $\|\cdot\|$. Let $\Delta$ be the unit disc in $\mathbb{C}$, and let $f: \Delta \rightarrow E$ be a holomorphic mapping. If there exists a point $\zeta_{0} \in \Delta$ such that $\left\|f\left(\zeta_{0}\right)\right\|=$ $\sup \{\|f(\zeta)\|: \zeta \in \Delta\}$, then $\|f(\zeta)\|$ is constant on $\Delta$.

\section{Proof of Main Theorem}

Let $\Delta$ be the unit disc in $\mathbb{C}$. We take a fixed boundary point $w \in \partial B$. Let $f(z)=\sum_{n=1}^{\infty} P_{n}(z)$ be the expansion of $f$ by $n$ homogeneous polynomials $P_{n}$ in a neighbourhood $V$ of 0 in $E$. Then we have $f(z)=z+\sum_{n=2}^{\infty} P_{n}(z)$. For $\zeta \in \Delta$,

$$
f(\zeta w)=\zeta w+\sum_{n=2}^{\infty} \zeta^{n} P_{n}(w) .
$$

Let $m \geq 2, m \in \mathbb{Z}$ be fixed. Let $a=\exp (2 \pi \sqrt{-1} / m)$. Then

$$
\begin{aligned}
\sum_{k=0}^{m-1} f\left(\zeta^{\frac{1}{m}} a^{k} w\right) & =\sum_{k=0}^{m-1}\left\{\zeta^{\frac{1}{m}} a^{k} w+\sum_{n=2}^{\infty}\left(\zeta^{\frac{1}{m}} a^{k}\right)^{n} P_{n}(w)\right\} \\
& =\zeta^{\frac{1}{m}}\left(\sum_{k=0}^{m-1} a^{k}\right) w+\sum_{n=2}^{\infty}\left(\sum_{k=0}^{m-1} a^{k n}\right) \zeta^{\frac{n}{m}} P_{n}(w) \\
& =m \sum_{j=1}^{\infty} \zeta^{j} P_{j m}(w) .
\end{aligned}
$$

This is holomorphic with respect to $\zeta \in \Delta$. Since $f(B)$ is convex, we have

$$
\frac{1}{m} \sum_{k=0}^{m-1} f\left(\zeta^{\frac{1}{m}} a^{k} w\right) \in f(B) .
$$


We set

$$
h(\zeta)=f^{-1}\left(\frac{1}{m} \sum_{k=0}^{m-1} f\left(\zeta^{\frac{1}{m}} a^{k} w\right)\right) .
$$

Then $h(\zeta)$ is holomorphic on $\Delta$. By the conditions on $f$,

$$
f^{-1}(z)=z+O\left(\|z\|^{2}\right)
$$

We have

$$
\begin{aligned}
h(\zeta) & =f^{-1}\left(\sum_{j=1}^{\infty} \zeta^{j} P_{j m}(w)\right) \\
& =\sum_{j=1}^{\infty} \zeta^{j} P_{j m}(w)+O\left(\left\|\sum_{j=1}^{\infty} \zeta^{j} P_{j m}(w)\right\|^{2}\right) \\
& =\zeta P_{m}(w)+O\left(|\zeta|^{2}\right) .
\end{aligned}
$$

Therefore $\frac{h(\zeta)}{\zeta}$ is a holomorphic mapping from $\Delta$ into $E$. If $\varepsilon>0$ is sufficiently small, $\frac{h(\zeta)}{\zeta}$ is continuous and holomorphic on the set $\{\zeta:|\zeta| \leq 1-\varepsilon\}$. Since $h(\Delta) \subset B$, by Theorem 2.1, we obtain

$$
\left\|\frac{h(\zeta)}{\zeta}\right\|<\frac{1}{1-\varepsilon}
$$

on $\{\zeta:|\zeta| \leq 1-\varepsilon\}$. Letting $\varepsilon$ tend to 0 , we have

$$
\left\|P_{m}(w)\right\|=\left\|\left.\frac{h(\zeta)}{\zeta}\right|_{\zeta=0}\right\| \leq 1
$$


for all $m \geq 2$. Then we have

$$
\begin{aligned}
\|f(\zeta w)\| & \leq\|\zeta w\|+\left\|\sum_{n=2}^{\infty} \zeta^{n} P_{n}(w)\right\| \\
& \leq|\zeta|+\sum_{n=2}^{\infty}|\zeta|^{n} \\
& =\frac{|\zeta|}{1-|\zeta|} \\
& =\frac{\|\zeta w\|}{1-\|\zeta w\|} .
\end{aligned}
$$

and the proof of the Main Theorem is complete.

Let $D$ be a bounded convex balanced domain in a complex Banach space $E$. The Minkowski function $N_{D}(z)$ of $D$ is defined by

$$
N_{D}(z)=\inf \{\alpha>0: z \in \alpha D\}
$$

for all $z \in E$. Then $N_{D}(z)$ is a norm on $E$. By the Main Theorem, we obtain the following corollary.

Corollary Let $D$ be a bounded convex balanced domain in a complex Banach space $E$. let $f: D \rightarrow E$ be a biholomorphic convex mapping such that $f(0)=0$ and $d f(0)$ is identity. Then

$$
N_{D}(f(z)) \leq \frac{N_{D}(z)}{1-N_{D}(z)}
$$

for all $z \in D$.

\section{References}

[1] S. Dineen, Complex Analysis in Locally Convex Spaces. North-Holland Math. Studies 57, 1981.

[2] S. Dineen, The Schwarz Lemma. Oxford Mathematical Monographs, 1989.

[3] N. Dunford and J. Schwartz, Linear Operators, vol. 1. Interscience: New York, 1958. 
[4] C. H. FitzGerald and C. R. Thomas, Some bounds on convex mappings in several complex variables, Pacific J. Math. 165 (1994), 295-320.

[5] T. Franzoni and E. Vesentini, Holomorphic Maps and Invariant Distances. North-Holland Math. Studies 40, 1980.

[6] S. Gong, Biholomorphic mappings in several complex variables, Contemporary Math. 142 (1993), 15-48.

[7] S. Gong and T. Liu, The growth theorem of biholomorphic convex mappings on $\mathbb{B}^{p}$, Chin. Quar. Jour. Math. 6 (1991), 78-82.

[8] H. Hamada, The growth theorem of convex mappings on the unit ball in $\mathbb{C}^{n}$, Proc. Amer. Math. Soc. 127 (1999), 1075-1077.

[9] M. Hervé, Analyticity in Infinite Dimensional Space. Walter de Gruyter, 1989.

[10] M. Jarnicki and P. Pflug, Invariant Distances and Metrics in Complex Analysis. Walter de Gruyter, 1993.

[11] T. Liu, The growth theorems, covering theorems and distortion theorems for biholomorphic mappings on classical domains, Thesis, University of Science and Technology of China, 1989.

[12] T. J. Suffridge, Biholomorphic mappings of the ball onto convex domains, Abstracts of papers presented to American Mathematical Society 11(66) (1990), 46.

Tatsuhiro Honda

Ariake National College of Technology

150 Higashihagio-machi

Omuta

Fukuoka

836-8585 Japan

e-mail: honda@ariake-nct.ac.jp 